\title{
Search for Dark Matter and Dark Sector at Belle II
}

\section{Thomas Hauth*}

Karlsruhe Institute of Technology

E-mail: Thomas.Hauthakit.edu

This contribution outlines the many possibilities to search for hints of dark matter and other dark sector particles with the Belle II detector. The Belle II experiment started data taking in 2018 and will resume in 2019 after all sub-detectors have been installed. Upgraded detector systems, especially new capabilities of the Level-1 hardware trigger, will enable the search for signatures which were challenging to perform at the previous generation B-Factories. This paper describes the search strategies for dark photons, axion-like particles and low mass dark matter candidates with Belle II and the projected limits that can be achieved after the first years of data taking.

XIV International Conference on Heavy Quarks and Leptons (HQL2018)

May 27- June 1, 2018

Yamagata Terrsa, Yamagata,Japan

${ }^{*}$ Speaker. 


\section{Introduction}

The Belle II experiment is operated at the asymmetric electron-positron collider SuperKEKB and recorded its first hadronic collision event at the end of April 2018. It is the successor of the Belle experiment, which ran from 1998 to 2010, and uses the same operating principle as the former KEKB collider to confirm the violation of $C P$ symmetry in the $B$ meson system. As a so-called B-factory, SuperKEKB operates on the $\Upsilon(4 S)$ resonance, which allows it to produce and record data from a large number of $B$ mesons. With the upgrade, the instantaneous luminosity will be increased by a factor 40 to $8 \times 10^{35} \mathrm{~cm}^{-2} \mathrm{~s}^{-1}$. Ultimately, Belle II is expected to record 50 times more collisions than Belle and to significantly increase the sensitivity of various searches and measurements [1]. Both the detector [2] and the software used to process the data will be upgraded to take advantage of technological developments and to be able to handle the greatly increased data rate.

The Belle II detector design continues to use many proven hardware options of Belle but is significantly upgraded in other areas. The innermost silicon-based tracking system VXD is a new development and consists of two layers of pixel sensors (PXD) and four layers of strip sensors (SVD). Followed by this is the central drift chamber (CDC) which uses the technologies proven in Belle and was sufficiently upgraded to support higher-occupancy environments. Next are the Time-of-Propagation (TOP) system in the barrel region and the Aerogel Ring Imaging Cherenkov counter (ARICH) system in the endcap area which provide particle identification. Finally, the electromagnetic calorimeter and the K-Long and Muon Detector (KLM) complete the instrumentation of Belle II.

\section{Motivation for Dark Matter and Dark Photon Searches}

Cosmological observations provide a clear indication for the existence of so-called dark matter, a form of matter that interacts via the gravitational force but not via the strong or electroweak force. While the phenomenological search for a dark matter candidate particle is pursued by many experiments, various models propose a range of new particles coupling to each other to build up the so-called dark sector. Multiple theoretical models exist and will be discussed in the following sections.

\section{Belle II's technical capabilities for Dark Matter and Dark Sector searches}

Many of the proposed signatures for physics beyond the standard model discussed in this paper are enabled by technological advances in the Belle II detector compared to the previous generation B-Factory detectors, Belle and BaBar.

Most dark matter and dark sector searches require one to properly trigger, measure and reconstruct individual charged or uncharged particles.

The Belle II trigger system consists of a hardware-based level-1 trigger (L1), which uses the input from the CDC, ECL, TOP, ARICH and KLM detectors[3]. Its primary purpose is to select $e^{+} e^{-}$annihilation events in the bunch crossings delivered by the SuperKEKB machine. Furthermore, the $\mathrm{L} 1$ will reduce the read-out rate of high cross section background processes like radiative 
Bhabha significantly and not trigger on tracks which do not originate from the interaction region, but rather are background induced. The L1 has a fixed latency of $5 \mu$ s and the L1 system has a maximum design output event rate of $30 \mathrm{kHz}$. This event data is then forwarded to the high-level trigger farm which is based on off-the-shelf x86-systems. Here, the data from sub-detectors are combined and a full event reconstruction is performed. With the higher precision of this reconstruction and by using the SVD hits in the track parameter estimate, a more fine-grained rejection of background or high cross section events is performed and the event output rate, which is written to permanent storage, is $10 \mathrm{kHz}$.

In contrast to Belle, the L1 of Belle II now has the ability to trigger on individual clusters in the electromagnetic calorimeter [4], a so-called single photon trigger. To facilitate this, the new level-1 trigger electronics also executes a clustering algorithm in the calorimeter as input to the trigger decision logic.

To veto large background processes in single photon searches, like $e^{+} e^{-} \rightarrow e^{+} e^{-} \gamma$, a good tracking coverage and reconstruction of charged tracks are required. To achieve this with the increased machine background of Belle II, a more fine grained tracking system is required to separate tracks from background noise. Belle II's CDC almost doubles the number of sense wires from 8,400 to 14,336 compared to Belle and the newly designed SVD has an excellent hit resolution of $8.2 \mu \mathrm{m}$ in p-strips and a high signal-to-noise ratio [5].

\section{Dark Photon}

One possible extension to the standard model (SM) are new U(1) gauge bosons, so-called dark photons $A^{\prime}$ which couple to the SM photon via kinetic mixing. The strength of this mixing is expressed by the parameter $\varepsilon$ which is defined as the mixing between the SM current $J_{S M}^{\mu}$ and the vector particle $V^{\mu}$ :

$$
\mathscr{L} \subset \varepsilon V_{\mu} J_{S M}^{\mu}
$$

Assuming the dark photon is produced together with one SM photon in $e^{+} e^{-} \rightarrow \mathrm{A}^{\prime} \gamma$, two decay channels for the dark photons exist and result in two distinct experimental signatures. In the dilepton mode, the $A^{\prime}$ decays into two SM leptons which results in one $\gamma$ and a pair of $l^{+} l^{-}$in the final state. A search for this decay channel was done with the BaBar dataset of $514 \mathrm{fb}^{-1}$ [6]. The other decay channel is the monophoton mode, where the $A^{\prime}$ decays into two light dark matter particles with $A^{\prime} \rightarrow \chi \bar{\chi}$. This channel is experimentally much more challenging to study as only one single photon can be detected with an energy of $E_{\gamma}=\left(E_{C M}^{2}-E_{A^{\prime}}^{2}\right) /\left(2 E_{C M}\right)$. Here, BaBar also performed a search, but only with a datset of $53 \mathrm{fb}^{-1}[6]$ with a special photon trigger.

Belle II will improve on the existing searches by BaBar and other experiments. Therefore, the L1 single photon trigger will be used during the regular data taking to increase the available dataset for dark photon searches. Primary backgrounds which need to be considered for such a trigger are radiative Bhabha processes $e^{+} e^{-} \rightarrow \gamma e^{+} e^{-}$where only the photon is in the acceptance of the detector. Trigger studies with the full background estimation have been performed and we expect a rate of $9 \mathrm{kHz}$ from this $\mathrm{L} 1$ trigger line. The challenges for the offline reconstruction are to achieve a good track finding efficiency over the full detector acceptance in order to reject radiative Bhabha processes. Furthermore, measurements from Belle II's outer KLM system can be used to veto $e^{+} e^{-} \rightarrow \gamma \gamma$ events where one photon was not detected by the electromagnetic calorimeter. 
Figures 1a and 1b show the expected limits for Belle II's dark photon search in the monophoton and dilepton channels for different sizes of the Belle II dataset. Especially in the monophoton channel, setting new upper limits is possible quickly after starting data taking at the design luminosity.
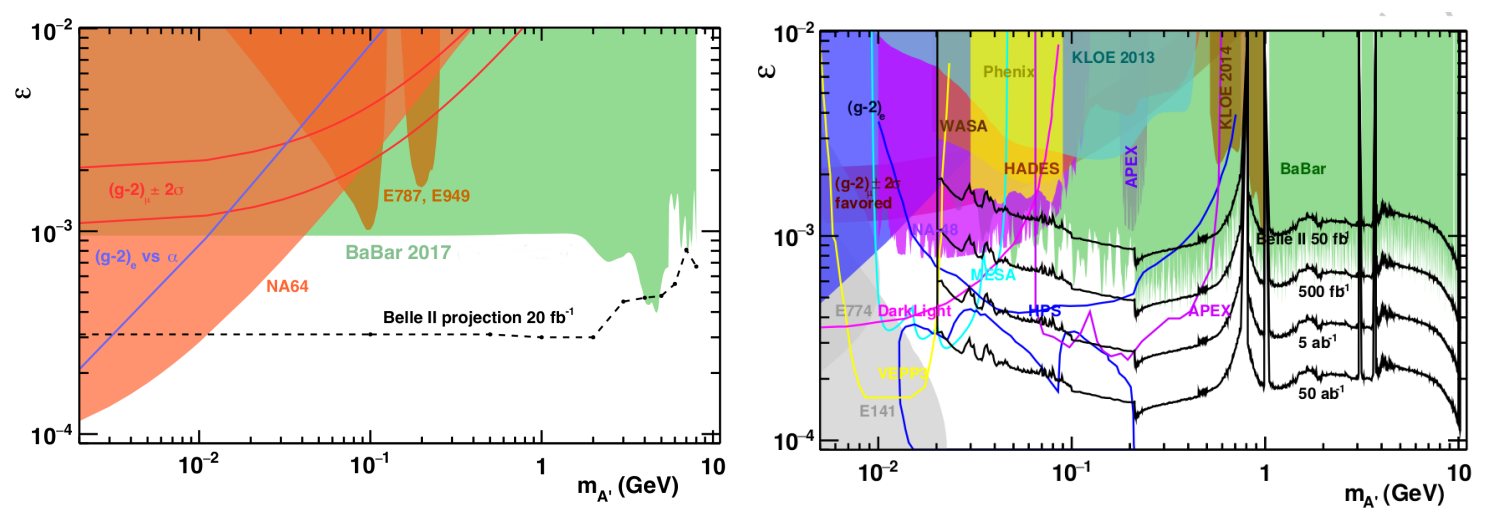

a) Projected upper limits on the kinematic b) Projected upper limits on the kinematic mixing parameter $\varepsilon$ between the dark pho- mixing parameter $\varepsilon$ between the dark photon $A^{\prime}$ and the SM for the process $e^{+} e^{-} \rightarrow$ ton $A^{\prime}$ and the SM for the process $e^{+} e^{-} \rightarrow$ $\gamma A^{\prime}\left(\rightarrow\right.$ invisible) for $20 \mathrm{fb}^{-1}$ as a function of $\gamma A^{\prime}(\rightarrow$ lepton lepton $)$ as a function of $A^{\prime}$ $A^{\prime}$ mass and mixing strength $\varepsilon$ [1]. $\quad$ mass and mixing strength $\varepsilon$ [1].

\section{Axion-like Particles}

Axion-like particles (ALP) can occur in multiple extension of the standard model, are pseudoscalar $\left(J^{P}=0^{-}\right)$and have separate couplings to fermions and the gauge bosons [1]. The most accessible ALP topology at Belle II is the case where the ALP couples to two SM photons.

Two experimental signatures need to be considered here. If the ALP is long lived, it will exit the Belle II detector volume before it decays further. Here, only one photon can be detected, which makes this analysis very similar to the one of the dark photon search discussed earlier. The other possibility is that the ALP decays to two photons, which will result in a total of three photons in the Belle II ECL. Figure 2 shows an overview in which regime an ALP discovery is possible depending on the ALP mass $m_{a}$ and coupling constant $g_{a \gamma}$. Invisible denotes that the ALP decays outside of the detector volume. In the Merged case, the ALP decays into two photons inside of Belle II but the individual photons cannot be resolved due to the limited spatial resolution of the ECL; in the Resolved regime, a proper separation of both photons is possible.

In this study a photon distance of two ECL clusters is required to put them in the Resolved category. This is a conservative estimate and can probably be improved with more advanced cluster reconstruction techniques.

Figure 3 shows the projected sensitivity ( $90 \% \mathrm{CL}$ ) of the photon and the hyper-charge coupling of the Belle II ALP search. Even for a modest dataset of $20 \mathrm{fb}^{-1}$, which can be accumulated in the first year of data taking with Belle II, the existing limits can be improved, especially for the $3 \gamma$ analysis. This can be further improved with the full Belle II dataset, which is expected to be $50 \mathrm{ab}^{-1}$. 


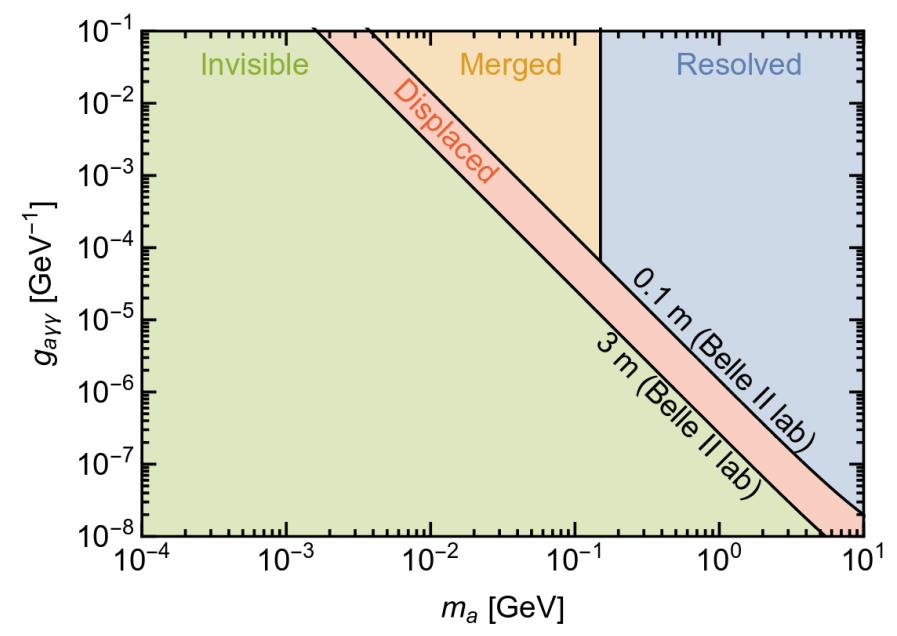

Figure 2: Illustration of the different kinematic regimes relevant for ALP decays into two photons with Belle II [7].
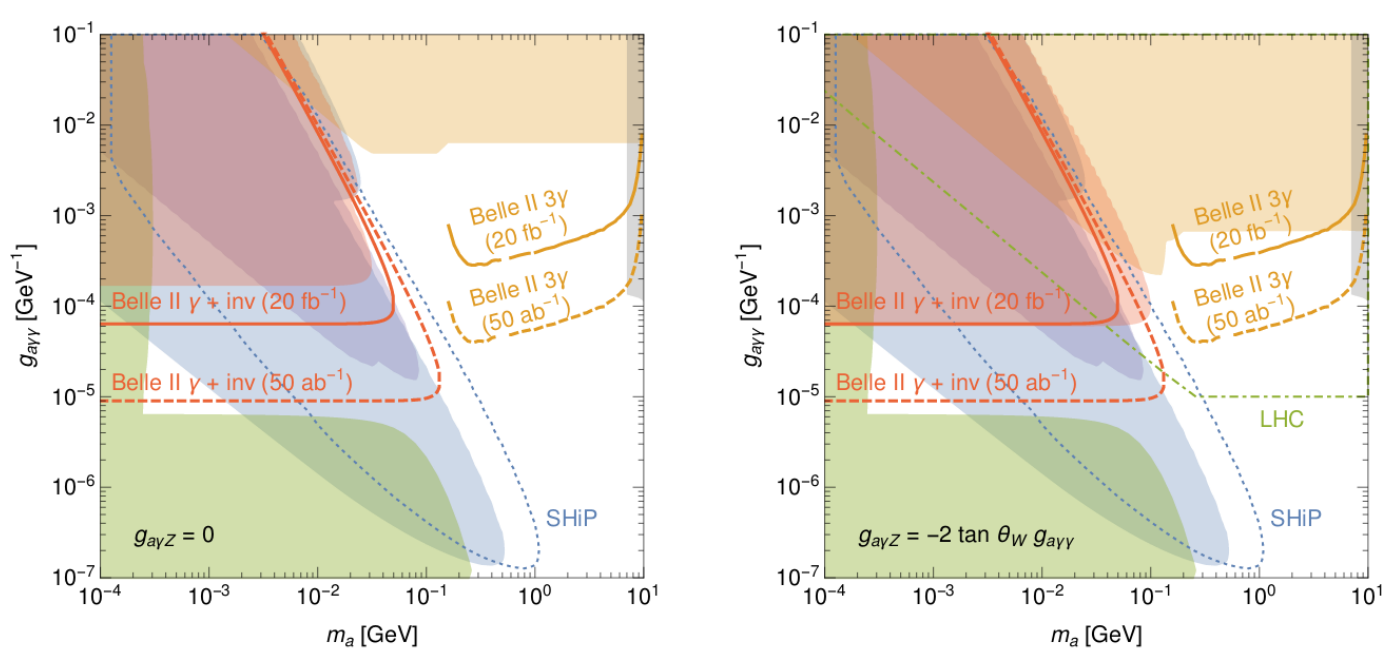

Figure 3: Projected Belle II sensitivity (90\% CL) compared to existing constraints on ALPs with photon coupling (left) and hypercharge coupling (right), as well as the projected sensitivities from SHiP and the LHC. [7].

\section{Low mass Dark Matter search}

In the $\mathrm{SM}$, invisible decays like $Y(1 S) \rightarrow v \bar{v}\left(\mathrm{BR} \approx 10^{-5}\right)$ exist, but they could be enhanced by an additional light dark matter particle. As the $Y(1 S)$ decay has no detectable final state products, transitions like $Y(3 S) \rightarrow \pi^{+} \pi^{-} Y(1 S)$ need to be exploited. The signal of $Y(1 S) \rightarrow$ invisible is an excess of events in the recoil mass for the two-pion system $M_{r}$ distribution at a mass equivalent to that of the $Y(1 S)$ of $9.460 \mathrm{GeV} / c^{2}$ [1]. Belle used a special trigger for $Y(3 S) \rightarrow \pi^{+} \pi^{-} Y(1 S)$ during the $Y(3 S)$ data taking, but no special trigger during the $Y(2 S)$ data taking. The ARGUS, CLEO, 
Belle and BaBar experiments have set the upper limit for the $\mathrm{BR}[Y(1 S) \rightarrow$ invisible $]$ at $3 \cdot 10^{-4}$ with a $90 \%$ confidence level.

Studies with the Belle II trigger simulation have shown that the special trigger conditions such as a single track trigger with $p_{t}>200 \mathrm{MeV} / \mathrm{c}^{2}$ (long track trigger) and an opening angle between the tracks in the $r-\phi$ plane larger than $30^{\circ}$ would allow for a trigger efficiency comparable to that of Belle (i.e. $85-90 \%$ ) in the process $Y(3 S) \rightarrow \pi^{+} \pi^{-} Y(1 S)$ and an efficiency of $30 \%$ for $Y(1 S) \rightarrow$ invisible [1].

The backgrounds for a $Y \rightarrow$ invisible search appear as distinct shapes in the $M_{r}$ distribution. The combinatorial background sources are mostly from processes like $e^{+} e^{-} \rightarrow e^{+} e^{-} X$, where both electrons escape detection and $X$ can be one of $X \rightarrow \pi^{+} \pi^{-}, \pi^{+} \pi^{-} \pi^{0}, \mu^{+} \mu^{-}$. Peaking background is present from two body $Y(1 S)$ decays where all decay products are outside of the detector acceptance.

Taking into account the expected contribution from the peaking background, one can expect a sensitivity at the $90 \%$ confidence level (statistical only) of $1.3 \times 10^{-5}$ for $\mathrm{BR}[Y(1 S) \rightarrow$ invisible $]$ when combining the various channels; this is comparable to the $\mathrm{SM}$ prediction of $\operatorname{BR}[Y(1 S) \rightarrow v v]$ $\approx 1.0 \times 10^{-5}[1]$.

\section{Conclusion}

The upgraded Belle II experiment will enable various avenues to perform searches for dark Matter and particles of the Dark Sector which have not been possible or limited by the amount of available events with the previous generation of B-Factory experiments. The new capabilities of the Belle II level 1 trigger to select single photon events will be essential to search for dark photons and axion-like particles and to surpass existing limits after a short time of data taking.

With the search for low mass dark matter candidates in $Y(1 S)$, Belle II can approach the SM branching fraction $Y(1 S) \rightarrow v v$ and thus will be able to detect a possible enhancement in this decay due to a new physics contribution.

\section{References}

[1] Belle II Collaboration and the B2TIP Theory Community, The Belle II Physics Book, to be submitted to PTEP (2018) [arXiv:1808.10567].

[2] Belle II Collaboration, Belle II Technical Design Report, arXiv:1011.0352.

[3] Y. Iwasaki, B. Cheon, E. Won, X. Gao, L. Macchiarulo, K. Nishimura et al., Level 1 trigger system for the Belle II experiment, IEEE Trans. Nucl. Sci. 58 (2011) 1807.

[4] T. Ferber, Towards First Physics at Belle II, Acta Phys. Polon. B46 (2015) 2285.

[5] Belle-II SVD Collaboration, Nakamura, Katsuro R. and others, The Belle II SVD detector, PoS Vertex2016 (2017) 012.

[6] BaBar Collaboration, Lees, J. P. and others, Search for a Dark Photon in $e^{+} e^{-}$Collisions at BaBar, Phys. Rev. Lett. 113 (2014) 201801 [arXiv: 1406.2980 ].

[7] M. J. Dolan, T. Ferber, C. Hearty, F. Kahlhoefer and K. Schmidt-Hoberg, Revised constraints and Belle II sensitivity for visible and invisible axion-like particles, Journal of High Energy Physics 12 (2017) 94 [arXiv:1709.00009]. 